\title{
Changes in Spine Alignment and Postural Balance After Breast Cancer Surgery: A Rehabilitative Point of View
}

\author{
Massimiliano Mangone, ${ }^{1, *}$ Andrea Bernetti, ${ }^{1}$ Francesco Agostini, ${ }^{1}$ Marco Paoloni, ${ }^{1}$ Francesco A. De Cicco, ${ }^{1}$
} Serena V. Capobianco, ${ }^{1}$ Arianna V. Bai, ${ }^{1}$ Adriana Bonifacino, ${ }^{2}$ Valter Santilli, ${ }^{1}$ and Teresa Paolucci ${ }^{3}$

\begin{abstract}
Breast cancer is the most common malignant tumor in female patients in developed countries. Recent articles indicate that one-sided mastectomy or minor breast surgery to treat breast cancer can have deleterious effects on posture and the musculoskeletal system. The purpose of this study was to investigate the alterations post-breast cancer surgery of the spine alignment associated to the balance not reported by the noninvasive instrumentation. We enrolled 30 women who had undergone treatment for breast cancer (BG) and were on a waiting-list for rehabilitation treatment and a control group of 30 healthy volunteer women (CG), matched by age and body mass index. The stabilometry was performed using a force platform (Kistler Instruments, Winterthur, Switzerland) test during quiet standing with closed-eyes (EC) and open-eyes (EO), recording the position of the center of pressure (CoP) for $51.2 \mathrm{sec}$. The stabilogram or the time plot of the two coordinates, $X$ and $Y$, of the CoP was obtained, which represent anteroposterior and midlateral balance. Spinal posture was measured using the Formetric-4D rasterstereographic system (DIERS, International GmbH, Schlangenbad, Germany), and thoracic kyphotic angle, lumbar lordotic angle, and surface trunk rotation were evaluated. Sixty participants were analyzed (CG:30; BG:30). For the spine rasterstereography a statistically significant difference was shown with regard to anterior-posterior flexion of the trunk major in BG; pelvic inclination and twist of half-pelvis decreased in BG; normalized lumbosacral inversion point decreased in BG; surface rotation major in BG; and lateral deviation major in BG. Compared with the values for the stabilometry test with $\mathrm{EO}$ and $E C$, a statistically significant difference was observed, respectively, for ellipse length ( $m m ; p=0.04$ ) and ellipse area $\left(\mathrm{mm}^{2} ; p=0.04\right)$ with EO and in ellipse area $\left(\mathrm{mm}^{2}\right)$ with EC $(p=0.05)$, increased in BG for both conditions. No difference was shown for CoP velocity and oscillations between the groups. Breast cancer survivors after prostheses or tissue expanders for mastectomy showed a spine's misalignment present both on the sagittal plane, both on the coronal and frontal plane, increased in BG regard to anterior-posterior flexion of the trunk, surface rotation, and lateral deviation. It is associated with greater energy expenditure for the postural balance control increased in BG with a major ellipse area in EO and EC conditions and major ellipse length in EC condition.
\end{abstract}

Keywords: balance; breast cancer; dysfunction; posture; rasterstereography; stabilometry

\footnotetext{
${ }^{1}$ Department of Anatomical and Histological Sciences, Legal Medicine and Orthopedics, Sapienza University of Rome, Rome, Italy.

${ }^{2}$ Breast Diagnosis and Treatment Unit, Sapienza University of Rome, Sant'Andrea Hospital, Rome, Italy.

${ }^{3}$ Umberto I University Hospital, Rome, Italy.

*Address correspondence to: Massimiliano Mangone, Eng, PhD, Department of Anatomical and Histological Sciences, Legal Medicine and Orthopedics, Sapienza University of Rome, Piazzale Aldo Moro 5, Rome 00185, Italy, E-mail: massimiliano.mangone@uniroma1.it
}

(c) Massimiliano Mangone, et al. 2019; Published by Mary Ann Liebert, Inc. This Open Access article is distributed under the terms of the Creative Commons License (http://creativecommons.org/licenses/by/4.0), which permits unrestricted use, distribution, and reproduction in any medium, provided the original work is properly cited. 


\section{Introduction}

Breast cancer is the most common malignant tumor in female patients in developed countries. ${ }^{1}$ Although treatment depends on the clinician indications, ${ }^{2,3}$ the primary therapeutic methodology consists of surgery mastectomy or breast-conserving therapy. Recent articles indicate that one-sided mastectomy or minor breast surgery to treat breast cancer can have deleterious effects on posture and the musculoskeletal system such as alterations in spine alignment, an increased thoracic kyphosis and upper limb dysfunctions, and decrease of shoulder joint angles at the operated side, ${ }^{4}$ but other studies appear to contradict these statements. ${ }^{1,5}$ After breast cancer surgery, patients have reduced physical participation in valuable activities contributed, more specifically, to explaining variability in depression. ${ }^{6}$ The patient tends to assume an attitude of closure, especially versus the presurgery "protective posture." In the early stages after surgery, this attitude is also due to modesty and shyness.

Women who undergo mastectomy alone, compared with women who undergo immediate breast reconstruction with abdominal flaps, for example, show differences in the vertical alignment of the trunk, with greater asymmetry between the acromion and greater trochanter, which can cause trunk rotation. ${ }^{7-11}$ The surgery technique seems to make a difference after breast cancer surgery. Also, lymphedema appears to worsen asymmetries and modifications in posture after mastectomy. ${ }^{12}$ Moreover, the literature is lacking in studies on posture balance after breast cancer surgery by stabilometry, instead a deterioration in bone strength and balance performance after breast cancer treatment can result in injurious falls: Fong et al. showed that Qigong may be a suitable exercise for improving the balance performance and balance self-efficacy. ${ }^{13}$ Postural stabilometry studies that have been performed after reductive or additive mammoplasty indicate. Mazzocchi et al. ${ }^{14,15}$ demonstrated that the head center of mass showed a significant variation on mediolateral direction, which indicated retro-positioning of the head. Some researchers believe that the increased weight of the breasts causes several spinal postural alterations, such as dorsal kyphosis and anterior shoulder dislodgement. ${ }^{16}$

Therefore, from these premises, it is possible to hypothesize that after mastectomy for breast cancer, postural spine alteration may occur especially on the sagittal plane together with a postural imbalance.

Then, the purpose of this study was to investigate whether breast cancer surgery results in spine postural alterations and postural imbalance to compare breast cancer patients with a population of healthy women. The primary outcome was the sagittal spine alignment regarding lumbar lordotic angle (LLA).

\section{Materials and Methods}

Study design and population

We conducted a case-control observational study consisting of a biomechanical evaluation of postural changes, as assessed within the sagittal and frontal planes, in female patients after surgical treatment for breast cancer and compared the postural balance control in the quiet stance. We enrolled a group of 30 women who had undergone treatment for breast cancer (BG) and were on a waiting list for rehabilitation treatment and a control group of 30 healthy volunteer women (CG), matched by age and body mass index (BMI). Participants were enrolled from January 1, 2018 to June 30, 2018 at the rehabilitation outpatient clinic of Policlinico Umberto I, Sapienza University of Rome (Italy).

All participants (patients and healthy controls) signed an informed consent form, after receiving detailed information about the study aims and procedures as per the Declaration of Helsinki. The protocol study was approved by the Ethics Committee of Sapienza University of Rome and was developed in accordance with the STROBE guidelines. ${ }^{17}$

The inclusion criteria for $\mathrm{BG}$ were as follows: total mastectomy performed within 12 months before recruitment (chronic phase) in waiting list for rehabilitative treatment, age from 18 to 60 years, BMI $<30$, no cognitive dysfunction, ${ }^{18}$ and use of breast prostheses or tissue expanders after mastectomy. The patients started the rehabilitative treatment within 1 month from the physiatric and postural test evaluations.

The exclusion criteria were as follows: conservative surgery, presence of lymphangitis or mastitis, surgical complications after the intervention, neurological deficits and complications, important shoulder joint problems before the intervention for breast cancer, previously diagnosed postural problems (scoliosis $>10^{\circ}$ Cobb angle), severe lymphedema and web axillary syndrome, ${ }^{11}$ visual problems that were not corrected by lenses, reconstruction with abdominal flaps or latissimus dorsi flaps, ${ }^{9-11,19}$ diabetes, hypertension that was not controlled by drugs, and antidepressant drug use.

The healthy group consisted of volunteer women who were in contact with our rehabilitation center. Their inclusion criteria were age from 18 to 60 years, $\mathrm{BMI}<30$, and no cognitive dysfunction. The exclusion 
criteria were postural problems, shoulder joint dysfunction, neurological or cognitive impairments, visual problems that were not corrected by lenses, oncological disease, rheumatological disorders, and pregnancy.

\section{Measurements}

All patients and healthy volunteers took part in a physiatrist visit to collect clinical data and measure the main postural parameters, to exclude scoliosis and other postural disorders. If necessary, an X-ray of the spine was obtained.

On the operated side, the physiatrist performed a clinical evaluation of the shoulder joint range of motion (ROM; 1. flexion, 2. extension, 3 . adduction, 4. abduction, 5. internal rotation, and 6. external rotation $)^{20}$ according to the scale of the Medical Research Council Manual Muscle Testing (MRC). ${ }^{21}$ A grade of $5 / 5$ on the MRC scale indicates that movement is possible against maximum resistance; $4 / 5$ indicates movement that is possible only against minimum resistance; $3 / 5$ indicates movement that is possible only against gravity; $2 / 5$ indicates movement that is possible only in the absence of gravity; $1 / 5$ indicates evidence of movement; and $0 / 5$ indicates no movement.

\section{Biomechanical evaluation}

Stabilometry assessment. Data were collected on a stabilometric platform (software Sway) to measure oscillations, sway area, length, and velocities of center of pressure with closed-eyes (EC) and open-eyes (EO). The stabilometry test was performed during quiet standing in both conditions (EC and EO) for $51.2 \mathrm{sec}$. After receiving information about the test procedure, the patients and healthy controls were instructed to stand erect, but not at attention, with their arms along the trunk, their feet at an angle of $\sim 30^{\circ}$ open toward the front, and their heels aligned along the mediolateral direction. All tests were performed by the same examiner; thus, the participants were supplied with the same instructions before each test. Three tests were conducted for each trial condition (EO and EC), and we have reported the average scores of the tests. In the EO condition, subjects fixated on a mark on a wall $1.5 \mathrm{~m}$ away at eye level. The test order, EO-EC or EC-EO, was randomized. To minimize external disturbances and cues for the test subjects, the environment was brightly lit naturally and quiet. ${ }^{22}$

Spine rasterstereography (Formetric). Spinal posture was measured using the Formetric $4 \mathrm{D}$ rasterstereographic system (DIERS, International GmbH, Schlangenbad, Germany). This device projects on the patient's back a series of parallel light stripes that are emitted by a slide projector. A three-dimensional reconstruction of the back surface is generated using triangulation equations, by transforming the stripes and their corresponding curvature into a scatter plot. The back surface curvature was evaluated, and concavity (right lumbar dimple or DR and left lumbar dimple or DL) and convexity (Vertebra prominens or VP) area were detected without reflective markers. ${ }^{23}$ Vertebral rotation in adolescent idiopathic scoliosis was calculated by radiograph and back surface analysis-based methods: correlation between the Raimondi method and rasterstereography. VP and DR and DL are specific back surface landmarks that are recognized automatically with a standard deviation (SD) of $\pm 1 \mathrm{~mm}$ for creating a Cartesian coordinate system. For Guidetti et al., ${ }^{24}$ no reflective markers were positioned on the patients.

Subjects were placed in a standing position at distance of $2 \mathrm{~m}$ from the system, barefoot in comfortable position, with their knees extended and their arms resting naturally alongside their hips. To standardize the subjects' positioning, a horizontal line was drawn on the floor to provide a reference for their heels (Fig. 1).

The following postural parameters were measured: anterior-posterior trunk flexion $(\mathrm{mm})$, lateral trunk flexion $(\mathrm{mm})$, pelvic inclination $(\mathrm{mm})$, twist of half-pelvis (degree), pelvis rotation (degree), apex of dorsal kyphosis $(\mathrm{mm})$, thoracolumbar inversion point (mm), apex of lumbar lordosis (mm), lumbosacral inversion point $(\mathrm{mm})$ normalized, cervical arrow (Stagnara; $\mathrm{mm}$ ), lumbar arrow (Stagnara; mm), thoracic kyphotic angle (max; degree), lumbar lordotic angle (max; degree), positive surface trunk rotation $(+\max =$ right; degree $)$, negative surface trunk rotation $(-\max =$ left; degree $)$, and the surface total trunk rotation at the end (amplitude $=$ total; degree).

The trunk inclination $(\mathrm{mm})$ is specified as the plumb line deviation from the VP to the midpoint between dimples (DM) along the sagittal plane; the kyphosis angle (degree) is measured as the angle between tangents of the spine curve, calculated at the points of cervicothoracic and thoracolumbar (ITL) inflexions; the lordotic angle (degree) is measured as the angle between tangents of the spine curve, calculated at the points of the ITL and lumbosacral junction inflexion; the Flèche cervicale and lombaire $(\mathrm{mm})$, or cervical arrow and lumbar arrow, are measured as the distances of the apex of the cervical and lumbar lordosis, respectively, from a virtual vertical plumb line; the pelvic tilt 


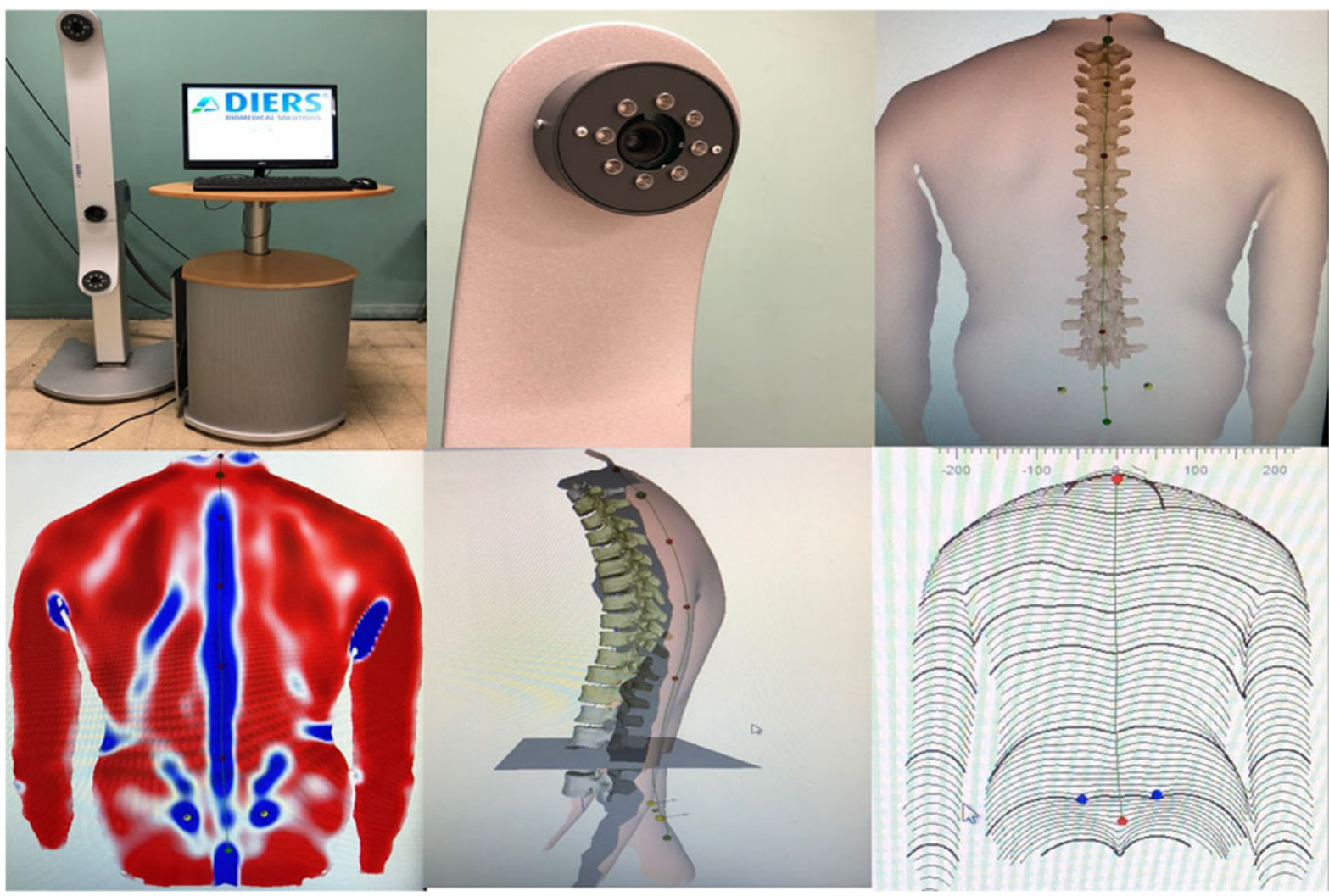

FIG. 1. Formetric.

(degree) is calculated as the arithmetic mean between the two angles that are formed by the perpendicular to the surface in the DR and DL to the vertical axis (pelvic torsion average).

\section{Scales: Functional assessment}

The Disabilities of Arm, Shoulder, and Hand Questionnaire $^{25}$ is a 30 -item, self-reported questionnaire that is designed to measure physical function and symptoms for disorders of the upper limb to quantify general disabilities that are related to the arm. The items are associated with the degree of difficulty in performing various functional activities due to arm, shoulder, or hand impairments (21 items); the severity of pain, activity-related pain, tingling, weakness, and stiffness (5 items); and the effect on social activities, work, and sleep and its psychological impact (4 items). The total score is converted to a scale from 0 to 100 $(100=$ greatest disability, $0=$ no disability $){ }^{26}$

The Constant-Murley score is one of the most widely used, valid, and reliable outcome measures for the assessment of the shoulder. ${ }^{27}$ This scoring system consists of subjective variables, such as pain (15 points), activities in daily living (10 points), and arm positioning (10 points), as well as objective variables, such as range of motion (40 points) and strength (25 points). The total score ranges from 0 to 100 , with a score of 100 indicating no limits.

Pain

The visual analog scale is a simple, robust, sensitive, and reproducible instrument that enables patients to express their pain intensity as a numerical value from 0 to 10. Patients are asked to associate the severity of their upper limb pain on the side of surgery with a position on a $10-\mathrm{cm}$ continuous line, marked "no pain" on one end and "worst pain" on the other. ${ }^{28,29}$

\section{Data analysis}

For the sample size calculation, the $G{ }^{*}$ Power Version 3.1.9.2 program was used. The difference between the two groups with respect to the LLA for spine rasterstereography was considered a primary parameter for postural outcome. ${ }^{30}$ 
The following values were considered for the lumbar lordotic angle with respect to the two groups: mean $(\mathrm{BG})=151.38 \quad( \pm 5.97)$, mean $(\mathrm{CG})=155.70 \quad( \pm 5.42)$; for a type 1 error $(\alpha)$ of $5 \%$, a type 2 error $(\beta)$ of $10 \%$, and a power level of 0.90 (using the $G{ }^{\star}$ Power Version 3.1.9.2), the required sample size was 30 participants per group. To allow for possible dropouts from the BG, we enrolled a total of 60 participants (30 patients in BG and 30 in CG).

The descriptive data were presented as means and SDs for all continuous variables. Variables were tested for normality using Shapiro-Wilk test; all the outcome measures were not normally distributed and so Mann-Whitney $U$ test was used to detect difference between groups. The significance level was set at $p<0.05$. Data were analyzed using MedCalc 12.2.1.0 (MedCalc Software).

\section{Results}

Data from 60 participants were analyzed: 30 participants in the CG (mean age $48 \pm 4.80$ years with a BMI of $24 \pm 1.20$ ) and 30 patients in the BG (mean age $50 \pm 5.94$ years with a BMI of $24 \pm 0.70$ ). The descriptive data of the sample, perfectly matched for age and BMI, are shown in Table 1.

For the spine rasterstereography, a statistically significant difference was shown between the two groups with regard to anterior-posterior flexion of the trunk

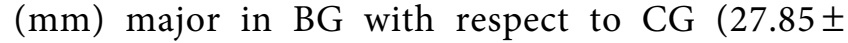
$32.60 \mathrm{~mm}$ for $B G$ vs. $20.02 \pm 10.94 \mathrm{~mm}$ for CG,

Table 1. Clinical Parameters

\begin{tabular}{|c|c|c|}
\hline Clinical parameters & $\begin{array}{l}\text { Control group } \\
\qquad(n=30)\end{array}$ & $\begin{array}{l}\text { Breast cancer } \\
\text { group }(n=30)\end{array}$ \\
\hline Age (mean $\pm S D)$ & $48 \pm 4.80$ & $50 \pm 5.94$ \\
\hline $\mathrm{BMI}($ mean $\pm \mathrm{SD})$ & $24 \pm 1.20$ & $24 \pm 0.7$ \\
\hline Married/common-law wife & $80 \%$ & $73 \%$ \\
\hline Working & Working $83 \%$ & Working $68 \%$ \\
\hline Not employed & Not employed $11 \%$ & Not employed $20 \%$ \\
\hline Or retired from work & Retired $6 \%$ & Retired $12 \%$ \\
\hline $\begin{array}{l}\text { At least a high school } \\
\text { education }\end{array}$ & $30 \%$ & $38 \%$ \\
\hline \multicolumn{3}{|l|}{ Clinical characteristics } \\
\hline Chemotherapy & - & $34.7 \%$ \\
\hline Radiotherapy & - & $55.4 \%$ \\
\hline Mild Lymphedema & - & $10 \%$ \\
\hline Time from surgery (months) & - & $4.65 \pm 3.30$ \\
\hline \multicolumn{3}{|c|}{ Scale scores for shoulder and upper limb disability } \\
\hline DASH scale & - & $58 \pm 14.4$ \\
\hline CMS & - & $61 \pm 8.62$ \\
\hline VAS & - & $2.54 \pm 2.46$ \\
\hline
\end{tabular}

Descriptive data (mean and SD) of the sample, matched for age and BMI. Control group (healthy) and breast cancer group (BG).

BMI, body mass index; CMS, Constant-Murley score; DASH, Disability of the Arm, Shoulder, and Hand; SD, standard deviation; VAS, visual analog scale. $p=0.001)$; pelvic inclination $(\mathrm{mm} ; 0.84 \pm 5.58 \mathrm{~mm}$ for BG vs. $-1.17 \pm 3.11$ for CG, $p=0.018$ ) decreased in BG with respect to CG; and twist of half-pelvis (degree) decreased in BG with respect to CG $\left(-0.04 \pm 2.99^{\circ}\right.$ for BG vs. $1.12 \pm 1.75^{\circ}$ for CG, $p=0.05$ ); normalized lumbosacral inversion point $(\mathrm{mm})$ decreased in BG with respect to CG $(-0.96 \pm 0.04 \mathrm{~mm}$ for BG vs. $0.99 \pm 0.02$ for CG, $p=0.005$ ); surface rotation (amplitude; degree) major in BG with respect to CG $\left(13.24 \pm 8.19^{\circ}\right.$ for BG vs. $11.79 \pm 3.92^{\circ}$ for CG, $\left.p=0.048\right)$; and lateral deviation $(\mathrm{mm})$ major in BG with respect to CG $(8.31 \pm 6.71 \mathrm{~mm}$ for BG vs. $5.60 \pm 3.38 \mathrm{~mm}$ for CG, $p=0.050$; Table 2). The minus sign indicates a rotation to the left side. Compared with the values for the stabilometry test with EO and EC, a statistically significant difference was observed, respectively, for ellipse length $(\mathrm{mm}$; $2364.39 \pm 287.84 \mathrm{~mm}$ for $\mathrm{BG}$ vs. $1505.01 \pm 330.29 \mathrm{~mm}$ for CG, $p=0.036)$ and ellipse area $\left(\mathrm{mm}^{2} ; 2789.40 \pm\right.$ $693.05 \mathrm{~mm}^{2}$ for BG vs. $2134.33 \pm 281.89 \mathrm{~mm}^{2}$ for CG, $p=0.042)$ with EO and in ellipse area $\left(\mathrm{mm}^{2}\right)$ with EC $\left(2948.07 \pm 1856.94 \mathrm{~mm}^{2}\right.$ for BG vs. $2028.67 \pm 538.19 \mathrm{~mm}^{2}$ for CG, $p=0.048$; Tables 3 and 4) increased in BG with respect to CG for both conditions (EO and EC).

\section{Discussion}

Our results demonstrate that postural alterations can be identified even after total mastectomy with external breast prostheses or tissue expanders. BG patients showed a greater limitation in sagittal spine alignment for anteriorposterior flexion of the trunk and lumbosacral inversion point more than a major pelvic inclination and twist of half-pelvis in favor of CG and an increase in surface rotation and lateral deviation. Also, in breast cancer patients, the postural control is carried out with greater energy expenditure considering the increasing of length and area with respect to the ellipse for the stabilometry evaluation.

Another study found that the amount of change in spinal alignment in postoperative breast cancer patients was significantly smaller with immediate breast reconstruction, compared with patients who received only unilateral mastectomy without reconstruction; thus, immediate breast reconstruction positively affects spinal alignment, leading to better posture and physical function. ${ }^{31}$ Our data are consistent with the literature and show that postural changes are evident even after surgical reconstruction during the first 12 months in chronic phase in breast cancer survivors: moreover, the postural alterations are not only on the sagittal plane of the column but also on the coronal plane and frontal one: it could be confirmed with the 
Table 2. Spine Rasterstereography: Trunk Values (Means and Standard Deviations)

\begin{tabular}{|c|c|c|c|}
\hline Spine rasterstereography: Trunk values & Healthy group, mean \pm SD & Breast cancer group, mean \pm SD & $p<0.05$ \\
\hline Anterior-posterior flexion (mm) & $20.02 \pm 10.94$ & $27.85 \pm 32.60$ & 0.001 \\
\hline Lateral flexion (mm) & $0.60 \pm 12.67$ & $-3.40 \pm 24.12$ & 0.378 \\
\hline Pelvic inclination (mm) & $-1.17 \pm 3.11$ & $0.84 \pm 5.58$ & 0.018 \\
\hline Twist of half-pelvis (degree) & $1.12 \pm 1.75$ & $-0.04 \pm 2.99$ & 0.005 \\
\hline Pelvis rotation (degree) & $0.22 \pm 3.51$ & $1.38 \pm 4.84$ & 0.703 \\
\hline Apex of dorsal kyphosis (mm) & $-0.36 \pm 0.04$ & $-0.33 \pm 0.67$ & 0.117 \\
\hline Thoracolumbar inversion point $(\mathrm{mm})$ & $-0.62 \pm 0.04$ & $-0.62 \pm 0.05$ & 0.564 \\
\hline Apex of lordosis (mm) & $-0.79 \pm 0.03$ & $-0.78 \pm 0.05$ & 0.071 \\
\hline Lumbosacral inversion point $(\mathrm{mm})$, normalized & $0.99 \pm 0.02$ & $-0.96 \pm 0.04$ & 0.005 \\
\hline Cervical arrow (Stagnara; $\mathrm{mm}$ ) & $52.98 \pm 13.62$ & $54.56 \pm 23.36$ & 0.113 \\
\hline Lumbar arrow (Stagnara; mm) & $35.92 \pm 12.04$ & $42.33 \pm 9.71$ & 0.250 \\
\hline Kyphotic angle (max; degree) & $52.53 \pm 8.25$ & $54.69 \pm 9.79$ & 0.274 \\
\hline Lordotic angle (max; degree) & $48.10 \pm 7.54$ & $51.45 \pm 11.47$ & 0.396 \\
\hline Surface rotation (+max; degree) & $5.10 \pm 3.98$ & $5.21 \pm 5.36$ & 0.871 \\
\hline Surface rotation (-max; degree) & $-6.68 \pm 2.48$ & $-8.02 \pm 5.64$ & 0.099 \\
\hline Surface rotation (amplitude; degree) & $11.79 \pm 3.92$ & $13.24 \pm 8.19$ & 0.048 \\
\hline Lateral deviation (mm) & $5.60 \pm 3.38$ & $8.31 \pm 6.71$ & 0.050 \\
\hline Surface rotation D4 (degree) & $3.12 \pm 2.28$ & $4.42 \pm 3.48$ & 0.511 \\
\hline Surface rotation D4 (mass; degree) & $-3.70 \pm 5.28$ & $-5.16 \pm 9.61$ & 0.195 \\
\hline Surface rotation D4 (amplitude; degree) & $5.26 \pm 3.30$ & $9.64 \pm 6.35$ & 0.448 \\
\hline
\end{tabular}

Variables were tested for normality using Shapiro-Wilk test; all the outcome measures were not normally distributed and so Mann-Whitney $U$-test was used to detect difference between groups.

hypothesis that this three-dimensional alteration also with pelvic angle modification in the BG is linked to a greater variability compared to the postural balance for the stabilometric test with respect to CG. It would be possible to deduce that breast cancer survivors engage specific postural adaptations after surgery that suggest to be not functional for the postural control.

Breast reconstruction after mastectomy has an impact on proper body posture ${ }^{32}$ but postural imbalance in breast cancer survivors may result a complex interaction between biomechanical alterations of posture and also, other factors as peripheral neuropathy for chemotherapy. ${ }^{33}$

We hypothesize that an increase in trunk flexion in the $\mathrm{BG}$, both with an alteration in pelvic inclination

Table 3. Stabilometry Parameters (Means and Standard Deviations), Open Eyes

\begin{tabular}{lccc}
\hline Parameters (EO) & Group & Mean \pm SD & $p<0.05$ \\
\hline Cop minimum swings (mm) & BG & $0.15 \pm 0.16$ & 0.377 \\
& CG & $0.10 \pm 0.08$ & \\
Cop maximum swings (mm) & BG & $17.42 \pm 6.31$ & 0.404 \\
& CG & $17.10 \pm 9.77$ & \\
Transversal axis (mm) & BG & $17.67 \pm 8.34$ & 0.073 \\
& CG & $14.84 \pm 5.53$ & \\
Longitudinal axis (mm) & BG & $29.64 \pm 9.57$ & 0.748 \\
Ellipse length (mm) & CG & $26.05 \pm 10.13$ & \\
& BG & $2364.39 \pm 287.84$ & 0.036 \\
Ellipse area $\left(\mathrm{mm}^{2}\right)$ & CG & $1505.01 \pm 330.29$ & \\
& BG & $2789.40 \pm 693.05$ & 0.042 \\
& CG & $2134.33 \pm 281.89$ & \\
\end{tabular}

Variables were tested for normality using Shapiro-Wilk test; all the outcome measures were not normally distributed and so Mann-Whitney $U$-test was used to detect difference between groups.

BG, breast cancer group; CG, control group of 30 healthy volunteer women. and twist of half-pelvis (Table 2), justifies the plus energy expenditure by BG for good postural control with respect to $C \mathrm{CG}^{34}$ In fact, for the stabilometry test, the BG results indicate greater ellipse length and ellipse area in the EO condition and higher ellipse area in the EC condition with respect to CG (Tables 3 and 4). Conversely, our results did not show a difference in the rotation of the trunk or lateral deviation with respect to the operated side in BG: some patients tend to have a closed posture toward the operated side due to shyness, ${ }^{35}$ pain, or tissue retraction with an increase in flexion of the trunk. We surmise that the alterations in other posture parameters, such as lumbosacral inversion point, lateral deviation, and surface rotation, during the spine rasterstereography test represent

Table 4. Stabilometry Parameters (Means and Standard Deviations), Closed Eyes

\begin{tabular}{lccc}
\hline Parameters (EC) & Group & Mean & $\boldsymbol{p}<\mathbf{0 . 0 5}$ \\
\hline Cop minimum swings & BG & $0.18 \pm 0.42$ & 0.116 \\
& CG & $0.08 \pm 0.06$ & \\
Cop maximum swings (mm) & BG & $20.92 \pm 9.57$ & 0.673 \\
& CG & $17.64 \pm 8.84$ & \\
Transversal axis (mm) & BG & $21.35 \pm 13.29$ & 0.670 \\
& CG & $19.40 \pm 14.29$ & \\
Longitudinal axis (mm) & BG & $33.98 \pm 13.77$ & 0.322 \\
Ellipse length (mm) & CG & $27.27 \pm 12.72$ & \\
Ellipse area (mm ${ }^{2}$ ) & BG & $1636.97 \pm 532.62$ & 0.615 \\
& CG & $1631.28 \pm 348.82$ & \\
& BG & $2948.07 \pm 1856.94$ & 0.048
\end{tabular}

Variables were tested for normality using Shapiro-Wilk test; all the outcome measures were not normally distributed and so Mann-Whitney $U$-test was used to detect difference between groups. 
compensation of the body due to the appearance of spine sagittal curves that each patient achieves for herself as an ergonomically optimal body position. ${ }^{36}$

It is important to emphasize that none of these patients had yet undertaken a postural or upper limb rehabilitation program at the beginning of our study. In the literature, ${ }^{37}$ researchers often describe the efficacy of early rehabilitation after surgery for breast cancer and preoperative fitness, ${ }^{38,39}$ but in medical practice, this approach still happens infrequently. In fact, our patients were sent to the physiatric consultation for rehabilitation by their oncologist or their surgeon, after an average of $4.65 \pm 3.30$ months since surgery often the delay is also due to the patient's inability to face the chemotherapy and/or radiotherapy path together with the rehabilitation.

Our results should encourage physicians to consider early and personalized rehabilitative programs, and in particular, with attention to postural disorders and postural imbalance. ${ }^{13}$

Above all, the programs should not only focus on the recovery of upper limb function but also include exercises for realignment of the trunk and exercises for improving postural control.

According to Barbosa Jde et al., ${ }^{40}$ the pelvis and trunk of women who have undergone quadrantectomy show better alignment compared with women who have been subjected to mastectomy but with little difference $\left(90^{\circ}\right.$ vs. $\left.91.3^{\circ}\right)$ in the short period after surgery. Also, the women who underwent surgery on the side breast had shoulder elevation and ipsilateral inclination of the trunk, but follow-up of this group after completion of treatment is needed to determine the long-term postural changes. ${ }^{40}$ Other groups, such as Saggini et al., ${ }^{41}$ reported a modification in posture and a significant increase in sway area after body mass alterations following mammoplasty, noting that posture and stabilometry data returned to equilibrium after 1 year. Their data suggest that posture control relies, at least in part, on feedforward and feedback strategies. ${ }^{41,42}$ After treatment of breast cancer, $82.3 \%$ of women demonstrate faulty body posture without a significant relationship between the quality of body posture and oncological treatment, ${ }^{43,44}$ but other groups, based on different data regarding radiotherapy, have reported more shoulder dysfunction in patients who undergo radiation therapy. ${ }^{45}$

\section{Weaknesses and strengths}

Our research expands on and enriches studies on posture and balance in women after breast surgery. There remain little data in the literature and virtually nothing on the use of specific tests, such as stabilometry and spine rasterstereography (with good objectivity). Above all, our results can be useful in developing more targeted rehabilitation programs that consider the recovery of not only the upper limb but also postural habit after breast cancer surgery. A limitation of the study is the lack of a postural assessment before surgery: for this reason it was considered a comparison group of healthy women. Our patients had never performed rehabilitative treatment and these data could be useful to investigate any changes that will be induced by the treatment itself at follow-up.

\section{Conclusion}

Breast cancer survivors after prostheses or tissue expanders for mastectomy showed an adaptation of body posture and postural control and they engaged in specific postural compensation. A misalignment of the spine is present both on the sagittal plane, both on the coronal and frontal plane increased in BG with regard to anterior-posterior flexion of the trunk, surface rotation, and lateral deviation. Moreover, it is associated with a greater energy expenditure for the postural balance control increased in BG with respect to CG with a major ellipse area in EO and EC conditions and major ellipse length in EC condition.

\section{Acknowledgments}

We thank all the women who agreed to participate in this research. Also, we appreciate the Breast Unit of Policlinico Umberto I and Prof. M. Monti, Director of the Complex Unit of General and Reconstructive Surgery of General Surgery-“F. Durante."

\section{Author Disclosure Statement}

The authors declare no conflict of interest and state that they had no source of funding for the research that has been reported.

\section{References}

1. Głowacka-Mrotek I, Sowa M, Nowikiewicz T, et al. Foot posture in female patients 5 years after breast-conserving surgery: a case-control study. Breast Cancer. 2018;25:325-333.

2. Veronesi U, Zucali R, Del Vecchio M. Conservative treatment of breast cancer with QU.A.R.T. Technique. World J Surg. 1985;9:676-681.

3. Veronesi U, Volterrani F, Luini A, et al. Quadrantectomy versus lumpectomy for small size breast cancer. Eur J Cancer. 1990;26:671-673.

4. Serel S, Tuzlalı ZY, Akkaya Z, et al. Physical effects of unilateral mastectomy on spine deformity. Clin Breast Cancer. 2017;17:29-33.

5. Hojan K, Manikowska F. Can the Weight of an external breast prosthesis influence trunk biomechanics during functional movement in postmastectomy women? Biomed Res Int. 2017;2017:9867694.

6. González-Fernández S, Fernández-Rodríguez C, Mota-Alonso MJ, et al. Emotional state and psychological flexibility in breast cancer survivors. Eur J Oncol Nurs. 2017;30:75-83. 
7. Crosible J, Kilbreath SL, Hollman L, et al. Scapulohumeral rhythm and associated spinal motion. Clin Biomech. 2008;23:184-192.

8. Crosible J, Kilbreath SL, Dylke E, et al. Effect of mastectomy on shoulder and spinal kinematics during bilateral upper-limb movement. Physical. 2010;90:679-692.

9. Yamamoto D, Tanaka Y, Tsubota Y, et al. Immediate breast reconstruction for breast cancer. Gan To Kagaku Ryoho. 2014;41:1892-1894.

10. Fischer JP, Fox JP, Nelson JA, et al. A longitudinal assessment of outcomes and healthcare resource utilization after immediate breast reconstruction-comparing implant- and autologous-based breast reconstruction. Ann Surg. 2015;262:692-699.

11. Atanes Mendes Peres AC, Dias de Oliveira Latorre MD, Yugo Maesaka J, et al. Body posture after mastectomy: comparison between immediate breast reconstruction versus mastectomy alone. Physiother Res Int. 2017;22:1

12. Haddad CA, Saad M, Perez Mdel C, et al. Assessment of posture and joint movements of the upper limbs of patients after mastectomy and lymphadenectomy. Einstein (Sao Paulo). 2013;11:426-434.

13. Fong SSM, Choi AWM, Luk WS, et al. Bone mineral density, balance performance, balance self-efficacy, and falls in breast cancer survivors with and without qigong training: an observational study. Integr Cancer Ther. 2018;17:124-130.

14. Mazzocchi M, Dessy LA, Di Ronza S, et al. A study of postural changes after breast reduction. Aesthetic Plast Surg. 2012;36:1311-1319.

15. Mazzocchi $M$, Dessy $L A$, lodice $P$, et al. A study of postural changes after breast augmentation. Aesthetic Plast Surg. 2012;36:570-577.

16. Nicoletti G, Passaro I, Malovini A, et al. Objective integrated assessment of functional outcomes in reduction mammaplasty. Plast Reconstr Surg Glob Open. 2013;1:e61.

17. Von Elm E, Altman DG, Egger M, et al.; STROBE Initiative. The Strengthening the Reporting of Observational Studies in Epidemiology (STROBE) statement: guidelines for reporting observational studies. Int J Surg. 2014;12:1495-1499.

18. Crum RM, Anthony JC, Bassett SS, et al. Population-based norms for the Mini-Mental State Examination by age and educational level. JAMA. 1993;269:2386-2391.

19. Albornoz CR, Matros $E$, Lee $C N$, et al. Bilateral mastectomy versus breastconserving surgery for early-stage breast cancer: the role of breast reconstruction. Plast Reconstr Surg. 2015;135:1518-1526.

20. Wingate L, Croghan I, Natarajan N, et al. Rehabilitation of the mastectomy patient: a randomized, blind, prospective study. Arch Phys Med Rehabil. 1989;70:21-24.

21. Ciesla N, Dinglas V, Fan E, et al. Manual muscle testing: a method of measuring extremity muscle strength applied to critically ill patients. J Vis Exp. 2011.;50:2632.

22. Scoppa F, Capra R, Gallamini M, et al. Clinical stabilometry standardization: basic definitions-acquisition interval-sampling frequency. Gait Posture. 2013;37:290-292.

23. Mangone $M$, Raimondi $P$, Paoloni $M$, et al. Vertebral rotation in adolescent idiopathic scoliosis calculated by radiograph and back surface analysisbased methods: correlation between the Raimondi method and rasterstereography. Eur Spine J. 2013;22:367-371.

24. Guidetti L, Bonavolonta V, Tito A, et al. Intra- and interday reliability of spine rasterstereography. Biomed Res Int. 2013;2013:745480.

25. Hudak PL, Amadio PC, Bombardier C. Development of an upper extremity outcome measure: the DASH (disabilities of the arm, shoulder and hand. The Upper Extremity Collaborative Group (UECG). Am J Industr Med. 1996;29:602-608.

26. Padua R, Padua L, Ceccarelli E, et al. Italian version of the disability of the arm, shoulder and hand (DASH) questionnaire: cross-cultural adaptation and validation. J Hand Surg. 2003;28:179-186.

27. Constant CR, Murley AHG. A clinical method of functional assessment of the shoulder. Clin Orthop Rel Res. 1987;217:160-164.

28. Davidson M, Keating JL. A comparison of five low back disability questionnaires: reliability and responsiveness. Phys Ther. 2002;82:8-24.

29. Strong J, Ashton R, Chant D. Pain intensity measurement in chronic low back pain. Clin J Pain. 1991;7:209-218.

30. Głowacka I, Nowikiewicz T, Hagner W, et al. Sagittal plane postural changes in female patients with breast cancer after different surgical techniques. Breast J. 2017;23:109-111.

31. Jeong JH, Choi B, Chang SY, et al. The effect of immediate breast reconstruction on thoracic spine alignment after unilateral mastectomy. Clin Breast Cancer. 2018;18:214-219.
32. Ciesla S, Polom K. The effect of immediate breast reconstruction with Becker-25 prosthesis on the preservation of proper body posture in patients after mastectomy. Eur J Surg Oncol. 2010;36:625-631.

33. Kneis S, Wehrle A, Freyler K, et al. Balance impairments and neuromuscular changes in breast cancer patients with chemotherapy-induced peripheral neuropathy. Clin Neurophysiol. 2016;127:1481-1490.

34. Ranavolo A, Don R, Cacchio A, et al. Comparison between kinematic and kinetic methods for computing the vertical displacement of the center of mass during human hopping at different frequencies. J Appl Biomech. 2008;24:271-279.

35. Oosterwijk S, Rotteveel M, Fischer AH, et al. Embodied emotion concepts: how generating words about pride and disappointment influences posture. Eur J Soc Psychol. 2009;39:457-466.

36. Le Huec JC, Saddiki R, Franke J, et al. Equilibrium of the human body and the gravity line: the basics. Eur Spine J. 2011;20(Suppl. 5):558-563.

37. Chrischilles EA, Riley D, Letuchy $E$, et al. Upper extremity disability and quality of life after breast cancer treatment in the Greater Plains Collaborative clinical research network. Breast Cancer Res Treat. 2019;175:675-689.

38. Liszka M, Samborski W. Assessment of biomechanical parameters of the shoulder joint at the operated side versus non-operated side in patients treated surgically for breast cancer. Rep Pract Oncol Radiother. 2018;23:378-383.

39. Yang A, Sokolof J, Gulati A. The effect of preoperative exercise on upper extremity recovery following breast cancer surgery: a systematic review. Int J Rehabil Res. 2018;41:189-196.

40. Barbosa Jde A, Amorim MH, Zandonade E, et al. Evalution of body posture in women with breast cancer. Rev Bras Ginecol Obstet. 2013;35:215-220.

41. Saggini R, lodice P, Saggini A, et al. Biomechanical modification and sense motor control of body posture after plastic surgery. Int J Immunopathol Pharmacol. 2012;25(1 Suppl.):1S-8S.

42. Terekhov Y. Stabilometry as a diagnostic tool in clinical medicine. Can Med Assoc J. 1976;115:631-633.

43. Hidding JT, Beurskens $\mathrm{CH}$, van der Wees PJ, et al. Treatment related impairments in arm and shoulder in patients with breast cancer: a systematic review. PLoS One. 2014;9:e96748.

44. Malicka I, Barczyk K, Hanuszkiewicz J, et al. Body posture of women after breast cancer treatment. Ortop Traumatol Rehabil. 2010;12:353-361.

45. Sugden EM, Reznavi M, Harrison JM, et al. Shoulder movement after the treatment of early stage breast cancer. Clin Oncol (R Coll Radiol). 1998;10: 173-181.

Cite this article as: Mangone $M$, Bernetti $A$, Agostini F, Paoloni M, De Cicco FA, Capobianco SV, Bai AV, Bonifacino A, Santilli V, Paolucci T (2019) Changes in spine alignment and postural balance after breast cancer surgery: a rehabilitative point of view, BioResearch Open Access 8:1, 121-128, DOI: 10.1089/biores.2018.0045. 